\title{
FRATTINI CLASSES OF SATURATED FORMATIONS OF FINITE GROUPS
}

\author{
Peter Förster
}

We study the following question: given any local formation of finite groups, do there exist maximal local subformations? An answer is given by providing a local definition of the intersection of all maximal local subformations.

Consider a closure operation $A$ on classes of finite groups (in the sense of [8]). A class $\mathcal{X}$ is called $A$-maximal in a class $\mathcal{Y}\left(\mathcal{X}<_{A} \mathcal{Y}\right)$, if

$$
\begin{aligned}
& \mathcal{X}=A \mathcal{X} \subset \mathcal{Y}=A \mathcal{Y}\left(\mathcal{X}<_{A} \mathcal{Y}\right) \text { and } \\
& \mathcal{X} \subset \mathcal{Z}=A \mathcal{Z} \subseteq \mathcal{Y} \Rightarrow \mathcal{Z}=\mathcal{Y}
\end{aligned}
$$

In Herzfeld [9] the question of existence of $A$-maximal subclasses of an $A$-closed class $\mathcal{Y}$ has been touched upon in the case that $A=Q R_{0}$ (the closure operation for formations) or $A=\left\langle Q R_{0}, E_{\Phi}\right\rangle$ (the closure operation for saturated formations) and for some related closure operations, taking an approach via the so-called $A$-Frattini class of an $A$-closed class, the class of all "non-generators". More interesting results, attributed to Bryce, have subsequently appeared in Herzfeld [10 (Appendix), 11, 12]. The main purpose of the present note is to solve two of the problems raised in [9]. First we show that a non-trivial formation which is saturated does not have maximal subformations; in fact we prove a slightly more general result, which will be needed for the intended application. Then we apply the lemma crucial to the proof of this first result to obtain a description of the "saturated Frattini class" of a saturated formation, from which one can deduce that saturated formations defined locally by saturated formations do not normally possess maximal saturated subformations.

Our notation will be taken from $[13,8,4,3]$. Moreover, $\mathcal{E}$ and $\mathcal{M}$ will denote the classes of finite and monolithic groups, respectively. In this note we will consider only finite groups. For some standard results in the theory of saturated formations the reader is referred to $[13,6,5,16]$.

Received 2 November 1989

Copyright Clearance Centre, Inc. Serial-fee code: 0004-9729/90 \$A2.00+0.00. 


\section{The relative $A$-Frattini class of an $A$-Closed class}

DEFinition 1.1: Let $A$ denote a closure operation on classes of finite groups and let $\mathcal{Y} \subseteq \mathcal{X}=A \mathcal{X} \subseteq \mathcal{E}$.

(a) The A-Frattini class of $\mathcal{X}$ modulo $\mathcal{Y}$ is defined by

$$
\Phi_{A}(\mathcal{X} \div \mathcal{Y})=\{G \in \mathcal{X} \mid \mathcal{Y} \subseteq \mathcal{Z} \subseteq \mathcal{X}=A(\{G\} \cup \mathcal{Z}) \Rightarrow \mathcal{X}=A \mathcal{Z}\}
$$

(b) The A-Frattini class of $\mathcal{X}$ is defined by

$$
\Phi_{A}(\mathcal{X})=\Phi_{A}(\mathcal{X} \div \emptyset)
$$

Clearly,

$$
\begin{aligned}
\Phi_{A}(\mathcal{X} \div \mathcal{Y}) & =\Phi_{A}(\mathcal{X} \div A \mathcal{Y}) \\
& =\{G \in \mathcal{X} \mid \mathcal{Y} \subseteq \mathcal{Z}=A \mathcal{Z} \subseteq \mathcal{X}=A(\{G\} \cup \mathcal{Z}) \Rightarrow \mathcal{X}=\mathcal{Z}\}
\end{aligned}
$$

We are really interested in $\Phi_{A}(\mathcal{X})$. The reason for defining $\Phi_{A}(\mathcal{X} \div \mathcal{Y})$ will become clear in Section 3, where $\Phi_{L}(\mathcal{F})$ for a saturated formation $\mathcal{F}$ is described (as a local formation) in terms of classes $\Phi_{Q R_{0}}\left(\mathcal{F}_{p} \div \varphi_{p}(\mathcal{F})\right)$ with $p$ a prime in the characteristic of $\mathcal{F}$ and the classes $\mathcal{F}_{p}$ and $\varphi_{p}(\mathcal{F})$ being certain formations associated with $\mathcal{F}$ in a natural manner. (Recall that $Q R_{0}$ denotes the closure operation for formations, and that $L=\left\langle Q R_{0}, E_{\Phi}\right\rangle$ is the closure operation for saturated - equivalently: local formations.)

We do not know whether the basic observation 1.3 below holds without the hypothesis that $A$ be finitary, that is to say that $A$ be required to satisfy

$$
G \in A \mathcal{X} \Rightarrow G \in A\left\{G_{1}, \ldots, G_{n}\right\} \text { for suitable } n \in \mathrm{N}, G_{i} \in \mathcal{X}(i=1, \ldots, n) .
$$

Anyway, our main interest is in the closure operations generated by some of $Q$ (quotient closure), $R_{0}$ (subdirect product closure), $E_{\Phi}$ (Frattini extensions). Since the closure operations listed here are obviously finitary, it suffices to make the following observation, a consequence of that fact that

$$
\left\langle A_{1}, \ldots, A_{n}\right\rangle \mathcal{X}=\bigcup_{i=0}^{\infty}\left(A_{1} \ldots A_{n}\right)^{i} \mathcal{X}
$$

for all classes $\mathcal{X}$ and all finitary closure operations $A_{1}, \ldots, A_{n}$.

REMARK 1.2. If $A_{1}, \ldots, A_{n}$ are finitary closure operations then so is their join $\left\langle A_{1}, \ldots, A_{n}\right\rangle$. 
Proposition 1.3. If $A$ is a finitary closure operation then for $\mathcal{Y}$ and $\mathcal{X}$ as in 1.1 we have:

$$
\Phi_{A}(\mathcal{X} \div \mathcal{Y})= \begin{cases}\bigcap\left\{\mathcal{Z} \subseteq \mathcal{E} \mid \mathcal{Y} \subseteq \mathcal{Z} \ll_{A} \mathcal{X}\right\}, & \text { if this set is non-empty } \\ \mathcal{X}, & \text { otherwise. }\end{cases}
$$

In particular, $\mathcal{Y} \subseteq \Phi_{A}(\mathcal{X} \div \mathcal{Y})=A \Phi_{A}(\mathcal{X} \div \mathcal{Y}) \subseteq \mathcal{X}$.

The proof of this proposition is a routine consequence of the following lemma (which, in fact, yields a more adequate proof than the one given in [11] for the case when $\mathcal{Y}=\emptyset$ ).

Lemma 1.4. If $A$ is finitary and $\mathcal{Y}<_{A} \mathcal{X}=A(\{G\} \cup \mathcal{Y})$ then there exists a class $\mathcal{Z} \lessdot_{A} \mathcal{X}$ such that $\mathcal{Y} \subseteq \mathcal{Z} \not \supset G$.

The proof of this lemma is a standard application of Zorn's Lemma, and relies heavily on $A$ being finitary: $\bigcup C$ is $A$-closed for any chain $C$ (with respect to $\subseteq$ ) of $A$-closed classes between $\mathcal{Y}$ and $\mathcal{X}$.

The above proposition can be applied in the following way: if one can show that $\Phi_{A}(\mathcal{X})$, the class of all non-generators of the $A$-closed class $\mathcal{X}$, comprises all groups in $\mathcal{X}$, then one can conclude that there are no maximal $A$-closed subclasses of $\mathcal{X}$. This is the sort of application we have in mind when studying $\Phi_{A}(\mathcal{X})$ for $A=Q R_{0}$ and $A=\left\langle Q R_{0}, E_{\Phi}\right\rangle$ in the remainder of this note. In the subsequent sections the above results will be used without further reference. We conclude this section with some elementary observations, the proofs of which will be left to the reader. For any closure operation $A$ and every class $\mathcal{X} \subseteq \mathcal{E}$ we put

$$
(A-1) \mathcal{X}=\{X \in \mathcal{X} \mid X \in A(\mathcal{X} \backslash\{X\})\} .
$$

REMARK 1.5 .

(a) $\Phi_{A}(\mathcal{X}) \subseteq(A-1) \mathcal{X}$ for all $A$-closed classes $\mathcal{X}$.

(b) $\mathcal{Y} \cap A(A-1) \mathcal{Y}=(A-1) \mathcal{Y}$ for any class $\mathcal{Y}$.

(c) $\Phi_{A}(\mathcal{X})=(A-1) \mathcal{X}$ for every $A$-closed class $\mathcal{X}$ and each unary closure operation $A$ such that $G \cong B$ whenever $A\{G\}=A\{H\}$.

(Examples of such closure operations are $Q, S, S_{n}, S_{w}, Q S, Q S_{w}, E_{\Phi}$.)

EXAMPLE 1.6: For a fixed class $\mathcal{R}$ and all classes $\mathcal{Y}$, define

$$
X_{\mathcal{R}} \mathcal{Y}=\{G \in \mathcal{E} \mid Q\{G\} \cap \mathcal{R} \subseteq \mathcal{Y}\}
$$

Then $X[R]=X_{\mathcal{R}} Q$ is a finitary closure operation satisfying

$$
\Phi_{X[\mathcal{R}]}(\mathcal{X})=X[\mathcal{R}](\mathcal{R} \cap(Q-1)(\mathcal{X} \cap \mathcal{R})) \text { whenever } \mathcal{X}=X[\mathcal{R}] \mathcal{X} \text {. }
$$

Note that taking $X[\mathcal{R}]$ with $\mathcal{R}=\mathcal{E}, \mathcal{R}=\mathcal{M} \cup\{1\}$, and $\mathcal{R}=\mathcal{P}$ (the class of all primitive groups) yields the closure operations for homomorphs, semiformations, and Schunck classes, respectively. 


\section{ThE $Q R_{0}$-Frattini ClASS OF A FORMATION}

LEMMA 2.1. If $\mathcal{F}$ is a formation then so is

$$
\mathcal{F}_{\mathbf{S}}=\left\{G \in \mathcal{E} \mid G^{\mathcal{F}} \leqslant \mathbf{S}(G)\right\}=\{G \in \mathcal{E} \mid G / \mathbf{S}(G) \in \mathcal{F}\}
$$

(Recall that $\mathbf{S}(G)$ denotes the socle of $G$, the product of all minimal normal subgroups of $G$. Further, $G^{\mathcal{F}}$ is the $\mathcal{F}$-residual of $G$, the smallest normal subgroup $N$ such that $G / N \in \mathcal{F}$.)

Proof: Clearly, $Q \mathcal{F}_{\mathbf{S}}=\mathcal{F}_{\mathbf{S}}$. Therefore $R_{0} \mathcal{F}_{\mathbf{S}}=\mathcal{F}_{\mathbf{S}}$ will follow from

$\left(^{*}\right) \quad M_{i}$ minimal normal in $G, G / M_{i} \in \mathcal{F}_{\mathbf{S}}(i=1,2), M_{1} \neq M_{2} \Rightarrow G \in \mathcal{F}_{\mathrm{S}}$. Now if $M_{i} \not G^{\mathcal{F}}$ for some $i$, then $G^{\mathcal{F}} \leqslant \mathbf{S}(G)$ is immediate from

$$
G^{\mathcal{F}} \cong G^{\mathcal{F}} M_{i} / M_{i}=\left(G / M_{i}\right)^{\mathcal{F}} \leqslant \mathbf{S}\left(G / M_{i}\right),
$$

whilst in the case when $M_{i} \times M_{2} \leqslant G^{\mathcal{F}}$ the same conclusion is obtained by using a similar argument.

We can now give a short proof of a result due to Bryce (namely, [12, 3.2]). Incidentally, it is worth observing that 3.1 and 3.6 from the same paper can actually be obtained as trivial consequences of this result.

Proposition 2.2. For any formation $\mathcal{F}$,

(a) $Q R_{0}(Q-1)(\mathcal{F} \cap \mathcal{M}) \subseteq \Phi_{Q R_{0}}(\mathcal{F})$;

in particular, for any $G \in \mathcal{F}$, we have

(b) $G^{\Phi_{Q R_{0}}(\mathcal{F})} \leqslant \mathbf{S}(G)$ (that is, $\mathcal{F} \subseteq \Phi_{Q R_{0}}(\mathcal{F})_{S}$ ), provided only that $\mathcal{F} \supset\{1\}$.

Proof: (a) It suffices to show that $X / \mathbf{S}(X) \in \mathcal{H}$ whenever $X \in \mathcal{F} \cap \mathcal{M}$ and $\mathcal{H} \ll_{Q R_{0}} \mathcal{F}$. If $X / S(X) \notin \mathcal{H}$ for some such $X$, then $X \notin \mathcal{H}_{S}$, yielding the following contradiction:

$$
\mathcal{H}<_{Q R_{0}} \mathcal{H}_{\mathbf{S}} \cap \mathcal{F}<Q R_{0} \mathcal{F}
$$

note that a group in $\mathcal{F} \backslash \mathcal{H}$ of least order belongs to $\mathcal{H}_{\mathbf{s}}$.

(b) By (a) any monolithic group in $\mathcal{F}$ is in $\Phi_{Q R_{0}}(\mathcal{F})_{S}$, so

$$
\mathcal{F} \subseteq \Phi_{Q R_{0}}(\mathcal{F})_{\mathbf{S}}
$$

For later applications we need a generalisation of a result from [12] attributed to Bryce. Note that $[12,3.7]$ is a special case of the latter result (namely, $[12,3.3]$ ). We employ the following notation for any class $\mathcal{H}$ of groups: $\chi(\mathcal{H})$ is the set of all primes 
$p$ such that the group of order $p$ is in $\mathcal{H}$. Further, if $p$ is a prime, the class $\mathcal{H}$ is called p-saturated if $G /\left(\Phi(G) \cap \mathrm{O}_{p}(G)\right) \in \mathcal{H}$ implies that $G \in \mathcal{H}$; in this case we write $\mathcal{H}=E_{\Phi}^{p} \mathcal{H}$. Note that if $\mathcal{H}$ is a saturated formation, then it is $p$-saturated for any prime $p$, and $\mathcal{H}$ also satisfies the further conditions required in the following corollary.

COROLLARY 2.3. If $\mathcal{H}=Q R_{0} \mathcal{H} \subseteq \mathcal{F}=Q R_{0} \mathcal{F}$ and

$$
\mathcal{H}=Q R_{0}\left\{G \in \mathcal{H} \cap \mathcal{M} \mid p \in \pi(\mathrm{S}(G)) \text { for a prime } p \text { with } \mathcal{H}=E_{\Phi}^{p} \mathcal{H}\right\}
$$

then

$$
\mathcal{H} \subseteq \Phi_{Q R_{0}}(\mathcal{F}) \text {. }
$$

In particular, a saturated formation $\mathcal{H} \neq\{1\}$ is contained in the $Q R_{0}$-Frattini class of any formation containing it.

Proof: Let $G \in \mathcal{H} \cap \mathcal{M}$ and assume that $p \in \pi(\mathbf{S}(G))$ for some prime $p$ such that $\mathcal{H}=E_{\Phi}^{p} \mathcal{H}$. Then $G$ is a proper quotient of the semidirect product $G \mathbf{P}\left(1_{G}^{p}\right)$, where $P\left(1_{G}^{p}\right)$ denotes the projective cover of the irreducible trivial $G F(p)[G]$-module $1_{G}^{p}$. Since $\mathbf{S}(G)$, and hence the monolithic group $G$, acts faithfully on the monolithic module $\mathbf{P}\left(1_{G}^{p}\right)$, it is clear that $G \mathbf{P}\left(1_{G}^{p}\right) \in \mathcal{M}$. Furthermore, arguing as in the proof of (Schmid's version of) Lubeseder's Theorem as given in [6], one can prove that $p$-saturation of the formation $\mathcal{H}$ implies that $\mathcal{H}$ contains the group $Z_{p}$ of order $p$ whenever it contains any group of order divisible by $p$. Now from the elementary fact that the radical of a $G F(p)[G]$-module $V$ lies in the Frattini subgroup of the semidirect product $G V$ we deduce that $G \mathbf{P}\left(1_{G}^{p}\right) \in E_{\Phi}^{p}\left\{G \times Z_{p}\right\} \subseteq \mathcal{H}$. Thus $G \in(Q-1)(\mathcal{F} \cap \mathcal{M})$, and the preceding proposition, together with our hypothesis on $\mathcal{H}$, yields that $\mathcal{H} \subseteq \Phi_{Q R_{0}}(\mathcal{F})$. $]$

From the following remark $([12,3.8])$ it is clear that the inclusion stated in 2.2a can be proper. (Note that a better proof of 2.4 can be obtained by using $2.2 \mathrm{a}$ together with a well-known result of Barnes and Kegel [1] (namely, [13, VI.7.21]) and the fact that for any formation $\mathcal{F}$ the class $\mathcal{F}_{\Phi}$ of all groups $G$ satisfying $A / B \nless \Phi(G / B)$ for any chief factor $A / B$ of $G$ such that $A \leqslant G^{\mathcal{F}}$ is also a formation.)

REMARK 2.4. Suppose that $G \in \mathcal{F} \cap \mathcal{M}\left(\mathcal{F}=Q R_{0} \mathcal{F}\right)$ satisfies $S(G) \leqslant \Phi(G)$. Then the semidirect product $\left(G / \mathbf{C}_{G}(\mathbf{S}(G))\right) \mathbf{S}(G)$ is in $\Phi_{Q R_{0}}(\mathcal{F})$.

Recall from [4] that for any chief factor $H / K$ of a group $G$, the monolithic primitive group $(H / K) * G$ associated with it is defined by the formula

$$
(H / K) * G= \begin{cases}\operatorname{Aut}_{G}(B / K)(H / K), & \text { if } H / K \text { is abelian, } \\ \operatorname{Aut}_{G}(B / K), & \text { otherwise. }\end{cases}
$$

Following [15] we say that the chief factor $H_{1} / K_{1}$ of a group $G_{1}$ is similar to the chief factor $H_{2} / K_{2}$ of $G_{2}$ if $\left(H_{1} / K_{1}\right) * G_{1} \cong\left(H_{2} / K_{2}\right) * G_{2}$. 
Given a formation $\mathcal{F}$ and a class $\mathcal{H}$ of monolithic primitive groups, it is straightforward from the Jordan-Hölder Theorem that the class $\mathcal{F}_{\mathcal{H}}$ of all groups $G$ satisfying $(H / K) * G \in \mathcal{H}$ for any chief factor $H / K$ of $G$ such that $B \leqslant G^{\mathcal{F}}$ is also a formation.

Corollary 2.5. Let $\mathcal{H} \varangle_{Q R_{0}} \mathcal{F}$ and $G_{i} \in(\mathcal{F} \backslash \mathcal{H}) \cap \mathcal{M}(i=1,2)$.

(a) $\mathrm{S}\left(G_{1}\right) * G_{1} \cong \mathrm{S}\left(G_{2}\right) * G_{2}$.

(b) $\Phi\left(G_{1}\right)=1 \Rightarrow G_{1} \cong G_{2}$.

(c) If $G_{1} \notin Q R_{0}\left\{G_{2}\right\}$, then there exists a $G \in \mathcal{H} \cap \mathcal{M}$ such that $\mathrm{S}(G) * G \cong S\left(G_{1}\right) * G_{1}$.

Proof: (a) Observe that (by 2.2a) $G_{i} / \mathrm{S}\left(G_{i}\right) \in \Phi_{Q R_{0}}(\mathcal{F}) \subseteq \mathcal{H}$ and consider the formations $\mathcal{H}_{i}=\mathcal{H}_{\left\{\mathbf{S}\left(G_{i}\right) * G_{i}\right\}}$ constructed above. Note that $G_{i} \in\left(\mathcal{F} \cap \mathcal{H}_{i}\right) \backslash \mathcal{H}$. In view of our hypothesis that $\mathcal{H} \lessdot Q R_{0} \mathcal{F}$ we must have that $\mathcal{F}=\mathcal{H}_{i} \cap \mathcal{F} \subseteq \mathcal{H}_{i}$. Consequently, $G_{3-i} \in \mathcal{H}_{i} \backslash \mathcal{H}$, and our claim follows.

(b) is immediate from (a) together with 2.4.

(c) Assume that $G_{1} \notin Q R_{0}\left\{G_{2}\right\}$. As

$$
G_{1} \in \mathcal{F}=Q R_{0}\left(\left\{G_{2}\right\} \cup \mathcal{H}\right)=Q R_{0}(\mathcal{K} \cup \mathcal{H}), \mathcal{K}=Q R_{0}\left\{G_{2}\right\}
$$

we find $H \in R_{0}(\mathcal{K} \cup \mathcal{H})$ and $K \unlhd H$ such that $G_{1} \cong H / K$. Choose some $H$ of least order subject to these conditions. Then

$$
H^{\mathcal{H}} \cap H^{\mathcal{K}}=H^{\mathcal{H}} \cap K=B^{\mathcal{K}} \cap K=1 \text {, but } H^{\mathcal{H}} \neq 1 \neq \boldsymbol{H}^{\mathcal{K}} .
$$

Since $H / \mathbf{S}(H \div K) \cong G_{1} / \mathbf{S}\left(G_{1}\right) \in \mathcal{H}$, we conclude that

$$
\mathbf{S}(H \div K)=H^{\mathcal{H}} \times K .
$$

Similarly,

$$
\mathbf{S}(H \div K)=\left(H^{\mathcal{K}} \cap \mathbf{S}(H \div K)\right) \times K \text {. }
$$

Hence $\left[\left(H^{\mathcal{K}} \cap \mathbf{S}(H \div K)\right) \times H^{\mathcal{H}}\right] / H^{\mathcal{H}} \cong_{H} \mathbf{S}(H \div K) / K=\mathrm{S}(H / K)$ is a chief factor of $H / H^{\mathcal{H}} \in \mathcal{H}$ similar to $\mathrm{S}\left(G_{1}\right)$.

Our next result essentially says that $\Phi_{Q R_{0}}$ preserves inclusions. An application in Section 3, however, requires a more general statement:

PROPOSITION 2.6.

(a) If $\mathcal{F}$ and $\mathcal{H}$ are formations and $\mathcal{G}$ is the formation generated by $\mathcal{F}$ and $\mathcal{H}$ - thus $\mathcal{G}=Q R_{0}(\mathcal{F} \cup \mathcal{H})$ - then

$$
\mathcal{K} \longmapsto \mathcal{L}=Q R_{0}(\mathcal{K} \cup \mathcal{H}), \quad \mathcal{L} \longmapsto \mathcal{F} \cap \mathcal{L}
$$


define mutually inverse bijections between the class of all formations $\mathcal{K}$ such that $\mathcal{F} \cap \mathcal{H} \subseteq \mathcal{K} \subseteq \mathcal{F}$ and the class of all formations $\mathcal{L}$ such that $\mathcal{H} \subseteq \mathcal{L} \subseteq \mathcal{G}$.

(b) If $\mathcal{F}<Q R_{0} \mathcal{G}$ and $\mathcal{H}<_{Q R_{0}} \mathcal{G}$, then either $\mathcal{F} \subseteq \mathcal{H}$, or else $\mathcal{F} \cap \mathcal{H}<_{Q R_{0}} \mathcal{F}$.

(c) If the formations $\mathcal{A}, \mathcal{B}, \mathcal{F}, \mathcal{G}$ satisfy $\mathcal{A} \subseteq B \cap \mathcal{F}$ and $B \cup \mathcal{F} \subseteq \mathcal{G}$, then

$$
\Phi_{Q R_{0}}(\mathcal{F} \div \mathcal{A}) \subseteq \Phi_{Q R_{0}}(\mathcal{G} \div \mathcal{B})
$$

Proof: (a) Let $\mathcal{K}$ and $\mathcal{L}$ be as in the statement of (a). We have to show that

$$
\mathcal{K}=\mathcal{F} \cap Q R_{0}(\mathcal{K} \cup \mathcal{H}) \text { and } \mathcal{L}=Q R_{0}([\mathcal{F} \cap \mathcal{L}] \cup \mathcal{H})
$$

Let $F \in \mathcal{F} \cap Q R_{0}(\mathcal{K} \cup \mathcal{H})$, so $F \cong G / N$ for some $G \in R_{0}(\mathcal{K} \cup \mathcal{H})$ and some $N \unlhd G$. We choose such $G$ of least order. As $G / N \in \mathcal{F} \supseteq \mathcal{K}$ for $R=G^{\mathcal{F}}$ we have that $R \leqslant N \cap G^{\mathcal{K}}$. Hence, from $G \in R_{0}(\mathcal{K} \cup \mathcal{H})$ we infer that

$$
(G / R)^{\mathcal{K}} \cap(G / R)^{\mathcal{H}}=G^{\mathcal{K}} R / R \cap G^{\mathcal{H}} R / R=\left(G^{\mathcal{K}} \cap G^{\mathcal{H}}\right) R / R=R / R,
$$

and in view of our minimal choice of $G$ we may assume that $R=1$; so $G \in \mathcal{F}$. It follows that $G / G^{\mathcal{H}} \in \mathcal{F} \cap \mathcal{H} \subseteq \mathcal{K}$ whence $G^{\mathcal{K}}=G^{\mathcal{K}} \cap G^{\mathcal{H}}=1$ and $G \in \mathcal{K}$. Now $\mathcal{K}=\mathcal{F} \cap Q R_{0}(\mathcal{K} \cup \mathcal{H})$ is clear.

Now let $L \in \mathcal{L}$. As $\mathcal{L} \subseteq \mathcal{G}$, by definition of $\mathcal{G}$ there exist $G \in R_{0}(\mathcal{F} \cup \mathcal{H})$ and $N \unlhd$ $G$ such that $L \cong G / N$. Again choosing $G$ of minimal order, we can apply an argument as above to deduce that without loss of generality $N \cap G^{\mathcal{H}}=1$ and hence $G \in R_{0} \mathcal{L}=\mathcal{L}$. We conclude that $G / G^{\mathcal{F}} \in \mathcal{F} \cap \mathcal{L}$. Consequently, $L \in Q\{G\} \subseteq Q R_{0}([\mathcal{F} \cap \mathcal{L}] \cup \mathcal{H})$.

(b) is immediate from (a).

(c) We may clearly assume that $\Phi_{Q R_{0}}(\mathcal{G} \div \mathcal{B})$ is the intersection of all $\mathcal{H}$ such that $\mathcal{B} \subseteq \mathcal{H} \lessdot Q R_{0} \mathcal{G}$. By (b), any such $\mathcal{H}$ contains $\mathcal{F}$ or some maximal subformation of $\mathcal{F}$ containing $\mathcal{A}$. If $\mathcal{F} \subseteq \mathcal{H}$ for every such $\mathcal{H}$, then

$$
\Phi_{Q R_{0}}(\mathcal{F} \div \mathcal{A}) \subseteq \mathcal{F} \subseteq \bigcap\left\{\mathcal{H} \subseteq \mathcal{E} \mid \mathcal{B} \subseteq \mathcal{H}<_{Q R_{0}} \mathcal{G}\right\}
$$

holds trivially, while otherwise $\mathcal{A} \subseteq \mathcal{F} \cap \mathcal{B}$ implies that

$$
\begin{aligned}
\bigcap\left\{\mathcal{H} \mid \mathcal{B} \subseteq \mathcal{H} \ll_{Q R_{0}} \mathcal{G}\right\} & \supseteq \bigcap\left\{\mathcal{F} \cap \mathcal{H} \mid \mathcal{B} \subseteq \mathcal{H} \ll_{Q R_{0}} \mathcal{G}\right\} \\
& \supseteq \bigcap\left\{\mathcal{K} \subseteq \mathcal{E} \mid \mathcal{A} \subseteq \mathcal{K} \ll_{Q R_{0}} \mathcal{F}\right\}=\Phi_{Q R_{0}}(\mathcal{F} \div \mathcal{A}) .
\end{aligned}
$$

Note that the last part of this proposition is genuinely a statement concerning Frattini classes rather than maximal subclasses: if $\mathcal{H}{\ll Q R_{0}} \mathcal{F}<Q R_{0} \mathcal{G}$ then there need 
not exist a $\mathcal{K} \ll_{Q R_{0}} \mathcal{G}$ containing $\mathcal{H}$; for example take $\mathcal{G}=\mathcal{E}_{p} \mathcal{E}_{q}$ for two primes $p$ and $q$ and note that $\mathcal{G}$, being a saturated formation, does not have maximal subformations (compare 2.3), while $\mathcal{E}_{p}$, the class of all p-groups, is evidently a maximal subformation of $\mathcal{F}=\left\{Q \times P \mid Q\right.$ elementary abelian g-group, $\left.P \in \mathcal{E}_{p}\right\}$.

Moreover, if $\mathcal{H}<_{Q R_{0}} \mathcal{F}<_{Q R_{0}} \mathcal{G}$ then there need not exist a $\mathcal{K}<_{Q R_{0}} \mathcal{G}$ such that $\mathcal{H}=\mathcal{F} \cap \mathcal{K}$ even under the additional hypothesis that $\Phi_{Q R_{0}}(\mathcal{G}) \neq \mathcal{G}$ : indeed, from 2.2a and $2.5 \mathrm{~b}$ one obtains the chain

$$
0 \varangle_{Q R_{0}}\{1\} \lessdot_{Q R_{0}} Q R_{0}\left\{Z_{2}\right\} \lessdot_{Q R_{0}} Q R_{0}\left\{S_{3}\right\} \lessdot_{Q R_{0}} Q R_{0}\left\{S_{4}\right\}
$$

We conclude this section by deriving analogues to some results on varieties from [14]; see also Chapter 5 of [15]. It will be seen that by means of applying our observations in this section, these results are considerably easier to establish than the corresponding results on varietes. We begin with a formation-theoretical characterisation of the monolithic groups, which is analogous to (and actually a special case of) $[14,1.3]$.

Corollary 2.7. $G \in \mathcal{M} \Leftrightarrow G \notin Q R_{0}(Q-1)\{G\}$.

Proof: Any non-monolithic group $G$ is clearly in $Q R_{0}(Q-1)\{G\}$, while for monolithic groups $G, 2.2$ a gives that $Q R_{0}(Q-1)\{G\} \subseteq \Phi_{Q R_{0}}\left(Q R_{0}\{G\}\right) \not \supset G$.

Next recall from [2] that a group $G$ is called formation-critical, if

$$
G \notin Q R_{0}\left((Q S-1)\{G\} \cap Q R_{0}\{G\}\right) .
$$

The following result is analogous to $[14,1.2]$.

COROLLARY 2.8. If a monolithic group $G$ is not formation-critical then

$$
G \in Q R_{0}\left(Q(S-1)\{G\} \cap Q R_{0}\{G\}\right) \subseteq Q R_{0}(S-1)\{G\} .
$$

Proof: For any group $X$,

$$
(Q S-1)\{X\}=Q(S-1)\{X\} \cup(Q-1)\{X\} .
$$

Thus $(Q-1)\{G\} \subseteq \Phi_{Q R_{0}}\left(Q R_{0}\{G\}\right)$ and $|(Q-1)\{G\}|<\infty$ yield that

$$
\begin{aligned}
Q R_{0}\{G\} & =Q R_{0}\left(\left[Q(S-1)\{G\} \cap Q R_{0}\{G\}\right] \cup(Q-1)\{G\}\right) \\
& =Q R_{0}\left(Q(S-1)\{G\} \cap Q R_{0}\{G\}\right) .
\end{aligned}
$$

Our last result in this section, a consequence of the previous one, is a formationtheoretical version of $[14,1.4]$. 
COROLlaRY 2.9. If a monolithic group $G$ is not formation-critical then $G / \mathbf{S}(G) \in Q R_{0}\left(Q(S-1)\{G / \mathbf{S}(G)\} \cap Q R_{0}\{G\}\right)$.

Proof: By 2.8, $G \cong H / K$ where $K \unlhd H \in R_{0}\left(Q(S-1)\{G\} \cap Q R_{0}\{G\}\right)$. Let $B$ be chosen of least order with respect to these properties and consider normal subgroups $K_{i}$ of $B(i=1, \ldots, n)$ such that $H / K_{i} \cong X_{i} / Y_{i} \in Q R_{0}\{G\} \cap \mathcal{M}$ where $X_{i}<G$ and $\bigcap_{i=1}^{n} K_{i}=1$. Also assume that $\bigcap_{j \neq i=1}^{n} K_{i} \neq 1$ for $j=1, \ldots, n$. Put $L=\bigcap_{i=1}^{n} \mathbf{S}\left(H / K_{i}\right)$ and observe that $L=\mathrm{S}(H)$. Since $B / K_{j}$ is monolithic, the subgroup $S_{j}=\mathrm{S}\left(H / K_{j}\right) \cap$ $\left(\bigcap_{j \neq i=1}^{n} K_{i}\right)$ is minimal normal in $H$, and hence, by minimality of $H$, is not contained in $K$. Therefore

$$
\mathbf{S}(H / K) \cong_{H} S_{j} \cong_{H} \mathbf{S}\left(H / K_{j}\right) \text { for } j=1, \ldots, n .
$$

It follows that either $X_{j} \cap \mathbf{S}(G)=Y_{j} \cap \mathbf{S}(G)$ or else $\mathbf{S}\left(X_{j} / Y_{j}\right)=\mathbf{S}(G) Y_{j} / Y_{j}$. In either case,

$$
X_{j} / \mathbf{S}\left(X_{j} / Y_{j}\right) \in Q(S-1)\{G / S(G)\} \text { for } j=1, \ldots, n:
$$

note that $X_{i}<G$ implies that $X_{i} \mathbf{S}(G)<G$, as $\mathbf{S}(G) \leqslant \Phi(G)$ for a not formationcritical monolithic group $G$. Now the assertion is immediate from

$$
L=\mathbf{S}(H) \leqslant \mathbf{S}(H / K) \text {. }
$$

\section{The L-Frattini class of a local formation}

In what follows the closure operation $\left\langle Q R_{0}, E_{\Phi}\right\rangle$ for saturated formations will be denoted by $L$. By the Gaschütz-Lubeseder Theorem (compare [13, VI.7.5] and [6]), $\mathcal{F}=L \mathcal{F}$ if, and only if, $\mathcal{F}=L F(f)$ for some formation function $f$ (that is, a function $f$ which associates to each prime $p$ a formation $f(p))$ : recall that $L F(f)$, the local formation defined by $f$, is the class of all $G \in \mathcal{E}$ such that $\operatorname{Aut}_{G}(H / K) \in f(p)$ for each chief factor $H / K$ of $G$ and any $p \in \pi(H / K)$. The reader is referred to Section 3 of [5] and Section 2 of [7] for the relevant terminology (but should note that in the present paper "local" is used instead of " $\mathcal{X}_{0}$-local"). It will be convenient to employ the following notation: $\mathcal{F}_{p}$ and $\mathcal{F}^{p}$ are, respectively, the minimal and the full and integrated $p$-local definitions of the saturated formation $\mathcal{F}$; compare $[7,2.1]$. It is well known that for $p \in \chi(\mathcal{F})$,

$$
\begin{aligned}
\mathcal{F}_{p} & =Q R_{0}\left\{\operatorname{Aut}_{G}(H / K) \mid G \in \mathcal{F}, H / K \text { chief factor of } G, p \in \pi(H / K)\right\} \\
& =Q\left\{G / O_{p^{\prime} p}(G) \mid G \in \mathcal{F}\right\}, \text { and } \\
\mathcal{F}^{p} & =\mathcal{E}_{p} \mathcal{F}_{p}=Q\left\{G / O_{p^{\prime}}(G) \mid G \in \mathcal{F}\right\}
\end{aligned}
$$


where $\mathcal{E}_{p}$ denotes the class of all p-groups. Finally the reader should recall that any formation function $f$ subject to the conditions that

$\mathcal{F}_{p} \subseteq f(p) \subseteq \mathcal{F}^{p}$ for each $p \in \chi(\mathcal{F})$ and $f(p)=\emptyset$ for every other prime $p$ is a local definition of $\mathcal{F}$ (that is, $\mathcal{F}=L F(f)$ ).

To state our main result, we need the following definition:

$$
\begin{aligned}
& \varphi_{p}(\mathcal{F})=Q R_{0} \Psi_{p}(\mathcal{F}), \text { where } \\
& \Psi_{p}(\mathcal{F})=\left\{\operatorname{Aut}_{G}(H / K) \mid G \in \mathcal{F}_{q} \cap \mathcal{M} \text { for some } q \in \chi(\mathcal{F}) \backslash\{p\},\right. \\
& \quad H / K \text { chief factor of } G \text { with the properties that } \\
& p \in \pi(H / K) \text { and }\{\{p, q\} \subseteq \pi(S(G)) \Rightarrow K \neq 1]\} .
\end{aligned}
$$

Furthermore, we put

$$
\mathcal{F}_{1} \times \mathcal{F}_{2}=\left\{F_{1} \times F_{2} \mid F_{i} \in \mathcal{F}_{i}(i=1,2)\right\}
$$

provided that for some set $\pi$ of primes $\mathcal{F}_{1}$ consists of $\pi$-groups, while $\mathcal{F}_{2}$ consists of $\pi^{\prime}$-groups; and we use $\mathcal{N}, \mathcal{E}_{\pi}$ to denote, respectively, the classes of nilpotent and of $\pi$-groups ( $\pi$ a set of primes).

TheOREM 3.1. For any local formation $\mathcal{F}$ and any prime $p$,

$$
\Phi_{L}(\mathcal{F})_{p} \subseteq \Phi_{Q R_{0}}\left(\mathcal{F}_{p} \div \varphi_{p}(\mathcal{F})\right) \subseteq \Phi_{L}(\mathcal{F})^{p}
$$

hence $\Phi_{L}(\mathcal{F})=L F(g)$ where the formation function $g$ is defined by

$$
g(p)=\Phi_{Q R_{0}}\left(\mathcal{F}_{p} \div \varphi_{p}(\mathcal{F})\right) \text { for all primes } p
$$

In particular,

$$
\begin{array}{cc}
\chi\left(\Phi_{L}(\mathcal{F})\right)=\left\{p \in \chi(\mathcal{F}) \mid \varphi_{p}(\mathcal{F}) \neq \emptyset \text { or } \mathcal{F}_{p} \neq\{1\}\right\} \subseteq \chi(\mathcal{F}) \\
\text { and } & \Phi_{Q R_{0}}\left(\mathcal{F}_{p}\right) \subseteq \Phi_{L}(\mathcal{F})^{p} \supseteq \varphi_{p}(\mathcal{F}) \text { for all primes } p .
\end{array}
$$

Proof: We will proceed in several steps.

(1) $\chi\left(\Phi_{L}(\mathcal{F})\right) \subseteq \chi(\mathcal{F})$ :

Since both $\Phi_{L}(\mathcal{F})$ and $\mathcal{F}$ are local formations (see 1.3), this is trivial.

(2) For all $p \in \chi(\mathcal{F})$, the following implication holds:

$$
\Phi_{L}(\mathcal{F})_{p} \cap \mathcal{M} \subseteq \Phi_{Q R_{0}}\left(\mathcal{F}_{p} \div \varphi_{p}(\mathcal{F})\right) \Rightarrow \Phi_{L}(\mathcal{F})_{p} \subseteq \Phi_{Q R_{0}}\left(\mathcal{F}_{p} \div \varphi_{p}(\mathcal{F})\right):
$$


If $\Phi_{L}(\mathcal{F})_{p} \notin\{1\}$ then $\Phi_{L}(\mathcal{F})_{p}=Q R_{0}\left(\Phi_{L}(\mathcal{F})_{p} \cap \mathcal{M}\right)$, and this implication holds trivially. In the case when $\Phi_{L}(\mathcal{F})_{p} \subseteq\{1\}$, the implication could fail to hold only if $\Phi_{L}(\mathcal{F})_{p}=\{1\}$ and $\Phi_{Q R_{0}}\left(\mathcal{F}_{p} \div \varphi_{p}(\mathcal{F})\right)=\emptyset$, which would require that $\varphi_{p}(\mathcal{F})=\emptyset$ and $\mathcal{F}_{p}=\{1\}$. From the latter we would see that $\mathcal{F} \subseteq \mathcal{E}_{p^{\prime}} \mathcal{E}_{p}$, yet $\mathcal{F} \nsubseteq \mathcal{E}_{p^{\prime}}$, while the former would give that $\mathcal{F}_{r} \subseteq \mathcal{E}_{p^{\prime}}$ for all $r \in \chi(\mathcal{F}) \backslash\{p\}$. These conditions, however, are easily seen to require that

$$
\mathcal{F}=\mathcal{E}_{p} \times\left(\mathcal{F} \cap \mathcal{E}_{p^{\prime}}\right)
$$

Obviously, for each $\mathcal{F}$ admitting such a decomposition, we must have that $\mathcal{F} \cap \mathcal{E}_{p^{\prime}} \lessdot_{L} \mathcal{F}$, from which the contradiction that $\Phi_{L}(\mathcal{F}) \subseteq \mathcal{E}_{p^{\prime}}\left(\right.$ that is, $\left.\Phi_{L}(\mathcal{F})_{p}=\emptyset\right)$ emerges.

For reference in a subsequent proof we record that the above argument actually establishes the following for an arbitrary local formation $\mathcal{F}$ :

$\left(^{*}\right) \quad \varphi_{p}(\mathcal{F})=\emptyset, \quad \mathcal{F}_{p}=\{1\} \Rightarrow \mathcal{F}=\mathcal{E}_{p} \times\left(\mathcal{F} \cap \mathcal{E}_{p^{\prime}}\right), \quad \Phi_{L}(\mathcal{F})_{p}=\emptyset$.

(3) $\Phi_{L}(\mathcal{F})_{p} \subseteq \Phi_{Q R_{0}}\left(\mathcal{F}_{p} \div \varphi_{p}(\mathcal{F})\right)$ for all $p \in \chi(\mathcal{F})$ :

By (2), it will suffice to show that $G \in \Phi_{Q R_{0}}\left(\mathcal{F}_{p} \div \varphi_{p}(\mathcal{F})\right)$ for all monolithic groups $G$ in $\Phi_{L}(\mathcal{F})_{p}$. The (first) description of $\mathcal{F}_{p}$ stated before the theorem, applied to $\Phi_{L}(\mathcal{F})_{p}$ instead of $\mathcal{F}_{p}$, shows that without loss of generality we may assume that $O_{p}(G)=1$ : Recall that $O_{p}\left(\operatorname{Aut}_{z}(X / Y)\right)=1$ whenever $X / Y$ is a chief factor of a group $Z$ and $p$ a prime dividing $|X / Y|$, and note that any group $\operatorname{Aut}_{Z}(X / Y)$ with this property is a subdirect product of monolithic groups enjoying the same property. By way of contradiction, assume that $\varphi_{p}(\mathcal{F}) \subseteq \mathcal{G}<_{Q R_{0}} \mathcal{F}_{p}$, yet $G \notin \mathcal{G}$. Put $\mathcal{H}=L F(h)$, where

$$
h(r)= \begin{cases}\mathcal{G}, & \text { if } r=p \\ \mathcal{F}_{r}, & \text { otherwise. }\end{cases}
$$

As $\mathcal{H}_{p} \subseteq h(p)=\mathcal{G} \subset \mathcal{F}_{p}$ and $\mathcal{H}_{q} \subseteq h(q) \subseteq \mathcal{F}_{q}$ for all primes $q \neq p$, we must have that $\mathcal{H} \subset \mathcal{F}$. Moreover, $\mathcal{E}_{p} \mathcal{G} \subseteq L F(h)=\mathcal{H}$ follows from the definition of $h$ together with the inclusions $\mathcal{G} \subseteq \mathcal{F}_{p} \subseteq \mathcal{F}$.

Since $G$ is monolithic and $O_{p}(G)=1$, there exists an irreducible and faithful $G$-module $V$ over $G F(p)$. The semidirect product $G_{0}$ of $G$ and $V$ lies in $\mathcal{E}_{p} \Phi_{L}(\mathcal{F})_{p} \subseteq \Phi_{L}(\mathcal{F})$. Consider the local formation $\mathcal{K}=L\left(\left\{G_{0}\right\} \cup \mathcal{H}\right) \subseteq \mathcal{F}$. If $\mathcal{K}=\mathcal{F}$ then $\mathcal{F}=L\left(\left\{G_{0}\right\} \cup \mathcal{H}\right)=\mathcal{H}$ (contradicting our choice of $\mathcal{H}$ ) follows from the fact that $G_{0} \in \Phi_{L}(\mathcal{F})$. Thus, arguing by way of contradiction, it remains to verify that $\mathcal{K}=\mathcal{F}$.

First we claim:

$$
\mathcal{K}^{p}=\mathcal{F P}^{p} \text {, and hence } \mathcal{K}_{p}=\mathcal{F}_{p} .
$$

Indeed, $\mathcal{K}^{p} \subseteq \mathcal{F P}$ follows from $\mathcal{K} \subseteq \mathcal{F}$. The reverse inclusion, however, also holds, as $\mathcal{E}_{p} \mathcal{G} \subseteq \mathcal{H} \subseteq \mathcal{K}$ (that is, $\mathcal{E}_{p} \mathcal{G} \subseteq \mathcal{K}^{p}$ ) and $G \cong$ Aut $_{G_{0}}(V)$ for the $p$-chief factor $V$ of 
$G_{0} \in \mathcal{K}$ (that is $G \in \mathcal{K}_{p}$ and thus $\mathcal{E}_{p}\{G\} \subseteq \mathcal{K}^{p}$ ), while $G \notin \mathcal{G} \ll_{Q R_{0}} \mathcal{F}_{p}$ (that is, $\left.\mathcal{F P}^{\mathcal{P}}=\mathcal{E}_{p} \mathcal{F}_{p}=\mathcal{E}_{p} Q R_{0}(\{G\} \cup \mathcal{G}) \subseteq \mathcal{K}^{p}\right)$.

We have assumed that $\mathcal{K} \subset \mathcal{F}$, which implies that $\mathcal{K}_{q} \subset \mathcal{F}_{q}$ for at least one $q$ in $\chi(\mathcal{F})$, where $q \neq p$ by $\left(^{* *}\right)$. By means of the argument at the beginning of this part of the proof, and observing that (as a consequence of the definition of $h$ ) $\{1\}=\mathcal{H}_{q} \subseteq \mathcal{K}_{q} \subseteq \mathcal{F}_{q}$ in the case when $\mathcal{F}_{q}=\{1\}$, we find a group

$$
F \in\left(\mathcal{F}_{q} \backslash \mathcal{K}_{q}\right) \cap \mathcal{M} \text { such that } O_{q}(F)=1 .
$$

As $1 \neq F \in \mathcal{F}_{q} \backslash \mathcal{K}_{q} \subseteq \mathcal{F}_{q} \backslash \mathcal{H}_{q}$, whereas $h(r)=\mathcal{F}_{r}$ for all $r \neq p$, there must exist a chief factor $A / B$ of $F$ such that $p \in \pi(A / B)$ and

$$
\operatorname{Aut}_{F}(A / B) \notin h(p)=\mathcal{G} \supseteq \varphi_{p}(\mathcal{F}) \supseteq \Psi_{p}(\mathcal{F}):
$$

If all chief factors of $F$ of order divisible by $p$ are $h$-central, then, as $q \neq p$, all chief factors of the semidirect product $F W$ of the $\mathcal{F}$-group $F$ with an irreducible and faithful $F$-module $W$ over $G F(q)$ are $h$-central, that is to say, $F W$ is in $L F(h)=\mathcal{H}$, and the contradiction that $F \cong F W / W=F W / C_{F W}(W) \in \mathcal{H}_{q}$ against the choice of $F$ emerges.

Now, by definition of $\Psi_{p}(\mathcal{F})$, and as $A / B$ is a chief factor of $F \in \mathcal{F}_{q} \cap \mathcal{M}$ satisfying $q \neq p \in \pi(A / B)$, the just established fact that $\operatorname{Aut}_{F}(A / B) \notin \Psi_{p}(\mathcal{F})$ requires the following conditions to hold:

$$
\{p, q\} \subseteq \pi(\mathbf{S}(F)), B=1 \text { and } A=\mathbf{S}(F)
$$

So $F \in \mathcal{F}$ is a monolithic primitive group with non-abelian minimal normal subgroup $\mathbf{S}(F)$. From the fact that $p \in \pi(\mathbf{S}(F))$, and using $\left({ }^{* *}\right)$, we infer that $F \in \mathcal{F}_{p}=\mathcal{K}_{p}$. Now $F \in \mathcal{K}$ and, being a monolithic primitive group with non-abelian socle satisfying $q \in \pi(S(F))$, it must also belong to $\mathcal{K}_{q}$. This contradicts the choice of $F$.

(4) $\varphi_{p}(\mathcal{F}) \subseteq \Phi_{L}(\mathcal{F})_{p}$.

Consider $G \in \mathcal{F}_{q} \cap \mathcal{M}$ for some $q \in \chi(\mathcal{F}) \backslash\{p\}$, and let $H / K$ be a chief factor of $G$ such that $p|| H / K \mid$ and $K \neq 1$ in the case when $\{p, q\} \subseteq \pi(H / K)$. Putting

$$
G^{*}=(\boldsymbol{H} / K) * G
$$

we shall show that $G^{*} \in \Phi_{L}(\mathcal{F})$. Suppose that $\mathcal{H}=L \mathcal{H} \subseteq \mathcal{F}=L\left(\left\{G^{*}\right\} \cup \mathcal{H}\right)$. We shall check that $\mathcal{F}=\mathcal{H}$. To begin with, observe that

$$
\mathcal{F}_{q}=Q R_{0}\left(\left\{\bar{G} / \mathbf{O}_{q^{\prime} q}(G)\right\} \cup \mathcal{H}_{q}\right) \text { where } \bar{G}=\operatorname{Aut}_{G}(H / K) .
$$


The assumption that $\bar{G} / \mathbf{O}_{q^{\prime} q}(G) \cong G$ would require that $G \cong$ Aut $_{G}(B / K)$ and $O_{q^{\prime} q}(G)=1=\mathbf{C}_{G}(H / K)$; in particular, $K=1$ and $G$ would be a monolithic primitive group with non-abelian socle such that $\{p, q\} \subseteq \pi(\mathbf{S}(G))$. This contradicts the conditions imposed on $G$ and $H / K$. Therefore we must have that $\bar{G} / O_{q^{\prime} q}(G) \in(Q-1)\{G\}$ and may apply $2.2 \mathrm{a}$ to $G\left(\in \mathcal{F}_{q} \cap \mathcal{M}\right)$ to get that $\bar{G} / \mathrm{O}_{q^{\prime} q}(G) \in \Phi_{Q R_{0}}\left(\mathcal{F}_{q}\right)$. The above description of $\mathcal{F}_{q}$ allows us to conclude that $G \in \mathcal{F}_{q}=\mathcal{H}_{q} \subseteq \mathcal{H}$. Using the BarnesKegel result quoted previously, we deduce that $G^{*} \in \mathcal{H}$ and $\mathcal{F}=L\left(\left\{G^{*}\right\} \cup \mathcal{H}\right)=\mathcal{H}$.

(5) $\Phi_{Q R_{0}}\left(\mathcal{F}_{p} \div \varphi_{p}(\mathcal{F})\right) \subseteq \Phi_{L}(\mathcal{F})^{p}$, for all $p \in \chi(\mathcal{F})$ :

It suffices to verify the following inclusion:

${ }^{(* * *)} \quad G \in \Phi_{Q R_{0}}\left(\mathcal{F}_{p} \div \varphi_{p}(\mathcal{F})\right) \cap(\mathcal{M} \cup\{1\}) \Rightarrow G \in \Phi_{L}(\mathcal{F})^{p}$.

As $\Phi_{L}(\mathcal{F})^{p}$ is a formation containing $\mathcal{E}_{p} \Phi_{L}(\mathcal{F})^{p}$, without loss of generality $\mathrm{O}_{p}(G)=1$. Thus there exists an irreducible and faithful $G$-module $V$ over $G F(p)$. We aim at showing that the semidirect product $G_{0}=G V$ belongs to $\Phi_{L}(\mathcal{F})$, which will clearly imply that $G \in \Phi_{L}(\mathcal{F})_{p} \subseteq \Phi_{L}(\mathcal{F})^{p}$. So, assuming that $\mathcal{F}=L\left(\left\{G_{0}\right\} \cup \mathcal{H}\right)$ for some local formation $\mathcal{H}$, we have to deduce that $\mathcal{F}=\mathcal{H}$. By 1.4, we may also assume that $\mathcal{H} \lessdot_{L} \mathcal{F}$. Now, as $G=\mathbf{A u t}_{G_{0}}(V)$, we see that $\mathcal{F}_{p}=Q R_{0}\left(\{G\} \cup \mathcal{H}_{p}\right)$.

If $\varphi_{p}(\mathcal{F}) \subseteq \mathcal{H}_{p}$ then $\mathcal{F}_{p}=\mathcal{H}_{p}$ is obvious, for $G \in \Phi_{Q R_{0}}\left(\mathcal{F}_{p} \div \varphi_{p}(\mathcal{F})\right)$ and $\mathcal{F}_{p}=$ $Q R_{0}\left(\{G\} \cup \mathcal{H}_{p}\right)$. In particular, $G \in \mathcal{H}_{p}$ and $G_{0}=G V \in \mathcal{E}_{p} \mathcal{H}_{p} \subseteq \mathcal{H}$, yielding that $\mathcal{F}=L\left(\left\{G_{0}\right\} \cup \mathcal{H}\right)=\mathcal{H}$.

Now we may assume that there is some $F \in \varphi_{p}(\mathcal{F}) \backslash \mathcal{H}_{p}$. By definition of $\varphi_{p}(\mathcal{F})$ as $Q R_{0} \Psi_{p}(\mathcal{F})$ such $F$ can be chosen in $Q \Psi_{p}(\mathcal{F}) \cap(\mathcal{M} \cup\{1\})$ and subject to the condition that $O_{p}(F)=1$. Consider an irreducible and faithful module $W$ for $F$ over $G F(p)$ and form the semidirect product $F_{0}=F W$. Without loss of generality, $G \in Q R_{0}\left(\{F\} \cup \mathcal{H}_{p}\right):$ otherwise the inclusion $\mathcal{H}_{p} \subset Q R_{0}\left(\{F\} \cup \mathcal{H}_{p}\right) \subset$ $Q R_{0}\left(\{G\} \cup \mathcal{H}_{p}\right)=\mathcal{F}_{p}$ would give that $\mathcal{H} \subset \mathcal{K} \subset \mathcal{F}$ with $\mathcal{K}=L\left(\left\{F_{0}\right\} \cup \mathcal{H}\right)$, which contradicts our assumption that $\mathcal{H} \lessdot_{L} \mathcal{F}-$ note that $\mathcal{K}_{p}=Q R_{0}\left(\{F\} \cup \mathcal{H}_{p}\right)$ and $\mathcal{H}_{q} \subseteq \mathcal{K}_{q} \subseteq \mathcal{F}_{q}$ for all $q \neq p$. Putting $\mathcal{L}=L\left(\left\{F_{0}\right\} \cup \mathcal{H}\right)$, we have that

$$
G \in \mathcal{F}_{p}=Q R_{0}\left(\{G\} \cup \mathcal{H}_{p}\right) \subseteq Q R_{0}\left(\{\mathcal{F}\} \cup \mathcal{H}_{p}\right)=\mathcal{L}_{p}
$$

Consequently, $G_{0} \in \mathcal{L}$. Since $\mathcal{H} \subseteq \mathcal{L} \subseteq \mathcal{F}$ and $G_{0} \notin \mathcal{H} \ll_{L} \mathcal{F}$, it follows that $\mathcal{L}=L\left(\left\{G_{0}\right\} \cup \mathcal{H}\right)=\mathcal{F}$. This, in turn, shows that $\mathcal{F}=\mathcal{L}=L\left(\left\{F_{0}\right\} \cup \mathcal{H}\right)=\mathcal{H}$, for from (4) we know that $F_{0}=F W \in \mathcal{E}_{p} \Phi_{L}(\mathcal{F})_{p} \subseteq \Phi_{L}(\mathcal{F})$. This proves ${ }^{* * *}$ ), and hence (5).

The theorem now follows from (1), (3) and (5).

In order to determine $\Phi_{L}(\mathcal{F})$ for local formations $\mathcal{F}$ with saturated local definitions, we need the following simple observation.

LEMMa 3.2. Let $\mathcal{F}=L F(f)$ for a formation function $f$ with the property that 
$f(p)=E_{\Phi} f(p)$ for some $p \in \chi(\mathcal{F})$. Then

(i) $\mathcal{F P}^{p}=\mathcal{E}_{p}(\mathcal{F} \cap f(p))$ is saturated; and

(ii) $\mathcal{F}_{p}$ is $q$-saturated for any prime $q \neq p$.

Corollary 3.3. Let $\mathcal{F}=L F(f) \neq\{1\}$.

(a) Assume that $f(p)=E_{\Phi} f(p)$ for some $p \in \chi(\mathcal{F})$. Then

$$
\Phi_{L}(\mathcal{F})_{p}= \begin{cases}\mathcal{F}_{p}, & \text { if } \varphi_{p}(\mathcal{F}) \neq \emptyset \text { or } \mathcal{F}_{p} \neq\{1\} \\ \emptyset, & \text { otherwise. }\end{cases}
$$

Moreover, if $\varphi_{p}(\mathcal{F})=\emptyset$ and $\mathcal{F}_{p}=\{1\}$, then $\mathcal{F}=\mathcal{E}_{p} \times\left(\mathcal{F} \cap \mathcal{E}_{p^{\prime}}\right)$.

(b) Assume that $f(p)=E_{\Phi} f(p)$ for all $p \in \chi(\mathcal{F})$ and put

$$
\pi=\left\{p \in \chi(\mathcal{F}) \mid \varphi_{p}(\mathcal{F}) \neq \emptyset \text { or } \mathcal{F}_{p} \neq\{1\}\right\} \text { and } \rho=\chi(\mathcal{F}) \backslash \pi \text {. }
$$

Then

$$
\Phi_{L}(\mathcal{F})=\mathcal{F} \cap \mathcal{E}_{\pi}, \mathcal{F}=\left(\times_{p \in p} \mathcal{E}_{p}\right) \times\left(\mathcal{F} \cap \mathcal{E}_{\pi}\right),
$$

and the $\mathcal{H} \ll_{L} \mathcal{F}$ are precisely the classes $\mathcal{H}=\mathcal{F} \cap \mathcal{E}_{p^{\prime}}$ for some $p \in \rho$.

Proof: (a) First suppose that $\mathcal{F}_{p} \neq\{1\}$. Then in view of statement (ii) in 3.2, we can apply 2.3 to deduce that

$$
\mathcal{F}_{p}=Q R_{0}\left\{G \in \mathcal{F}_{p} \cap \mathcal{M} \mid \mathbf{S}(G) \nless \mathbf{O}_{p}(G)\right\} \subseteq \Phi_{Q R_{0}}\left(\mathcal{F}_{p}\right)
$$

Consequently, $\mathcal{F}_{p}=\Phi_{Q R_{0}}\left(\mathcal{F}_{p}\right)$, so from 3.1 we infer that

$$
\mathcal{F P}^{\mathcal{P}}=\mathcal{E}_{p} \mathcal{F}_{p}=\mathcal{E}_{p} \Phi_{Q R_{0}}\left(\mathcal{F}_{p}\right) \subseteq \mathcal{E}_{p} \Phi_{L}(\mathcal{F})^{p}=\Phi_{L}(\mathcal{F})^{p} \subseteq \mathcal{F P}
$$

This shows that $\Phi_{L}(\mathcal{F})^{p}=\mathcal{F}^{p}$ and $\Phi_{L}(\mathcal{F})_{p}=\mathcal{F}_{p}$.

Finally, let $\mathcal{F}_{p}=\{1\}$. In the case when $\varphi_{p}(\mathcal{F}) \neq \emptyset$ we obtain that $\Phi_{Q R_{0}}\left(\mathcal{F}_{p} \div \varphi_{p}(\mathcal{F})\right)=\Phi_{Q R_{0}}(\{1\} \div\{1\})=\{1\}$, and from 3.1 it is immediate that $\Phi_{L}(\mathcal{F})_{p}=\{1\}=\mathcal{F}_{p}$. Thus we may also assume that $\varphi_{p}(\mathcal{F})=\emptyset$. This situation, however, was dealt with in statement $\left(^{*}\right)$ from the proof of 3.1, where it was shown that this requires that

$$
\mathcal{F}=\mathcal{E}_{p} \times\left(\mathcal{F} \cap \mathcal{E}_{p^{\prime}}\right) \text { and } \Phi_{L}(\mathcal{F})_{p}=\emptyset
$$

(b) is easily derived from (a). 
For our next result, recall that $\mathcal{N}$ denotes the class of all nilpotent groups.

COROLLARY 3.4. Let $\mathcal{F}=L \mathcal{F} \subseteq \mathcal{N N}$. Then for all $p \in \chi(\mathcal{F})$, we have

$$
\Phi_{L}(\mathcal{F})_{p}= \begin{cases}\{1\}, & \text { if } \mathcal{F} \subseteq \mathcal{E}_{p^{\prime}} \mathcal{E}_{p} \text { and } \mathcal{F} \notin \mathcal{E}_{p} \mathcal{E}_{p^{\prime}}, \\ \Phi_{Q R_{0}}\left(\mathcal{F}_{p}\right), & \text { otherwise. }\end{cases}
$$

Proof: Since $\mathcal{F} \subseteq \mathcal{N} N$, we have that $\mathcal{F}_{p} \subseteq \mathcal{E}_{p^{\prime}}$, and so it is clear from 3.1 that $\Phi_{L}(\mathcal{F})_{p}=\Phi_{Q R_{0}}\left(\mathcal{F}_{p} \div \varphi_{p}(\mathcal{F})\right)$. Now the result follows, using arguments as above, and taking into account that $\varphi_{p}(\mathcal{F})=\Psi_{p}(\mathcal{F}) \subseteq\{1\}$ follows from the inclusion $\mathcal{F}_{q} \subseteq \mathcal{N}$ for all primes $q$.

COROLLARY 3.5. If $\mathcal{F}=L \mathcal{F} \subseteq \mathcal{G}=L \mathcal{G}$ then $\Phi_{L}(\mathcal{F}) \subseteq \Phi_{L}(\mathcal{G})$.

Proof: First observe that $\Psi_{p}(\mathcal{F}) \subseteq \Psi_{p}(\mathcal{G})$ for all $p \in \chi(\mathcal{F})$. Then apply 3.1 together with 2.6c.

Corollary 3.6. Let $\mathcal{F}=L \mathcal{F}$ and $G \in \mathcal{F}_{p} \cap \mathcal{M}, p \in \chi(\mathcal{F})$. Then

$$
G \in \Phi_{L}(\mathcal{F})
$$

provided that one of the following three conditions holds:

$$
\Phi(G) \neq 1, \mathbf{S}(G) \text { is abelian, or } p \nmid|\mathbf{S}(G)| \text {. }
$$

Proof: From 2.2a and 3.1 we get that $G / \mathrm{S}(G) \in \Phi_{Q R_{0}}\left(\mathcal{F}_{p}\right) \subseteq \Phi_{L}(\mathcal{F})^{p} \subseteq \Phi_{L}(\mathcal{F})$. Consequently, if $\Phi(G) \neq 1$ then $G \in E_{\Phi} \Phi_{L}(\mathcal{F})=\Phi_{L}(\mathcal{F})$.

Now we may assume that $\Phi(G)=1$. If $\mathbf{S}(G)$ is non-abelian then by hypothesis $p \notin \pi(\mathbf{S}(G))$. Hence for any prime $q$ dividing $|\mathbf{S}(G)|$ we have that $G \cong \mathbf{A u t}_{G}(\mathbf{S}(G)) \in$ $\varphi_{q}(\mathcal{F}) \subseteq \Phi_{L}(\mathcal{F})^{q} \subseteq \Phi_{L}(\mathcal{F})$. Finally, let $S(G)$ be an abelian $q$-group for some $q \in \chi(\mathcal{F})$. Without loss of generality, $q \neq p$ : otherwise, as $G / \mathbf{S}(G) \in \Phi_{Q R_{0}}\left(\mathcal{F}_{p}\right) \subseteq \Phi_{L}(\mathcal{F})^{p}$, we get that $G \in \mathcal{E}_{p} \Phi_{L}(\mathcal{F})^{p}=\Phi_{L}(\mathcal{F})^{p} \subseteq \Phi_{L}(\mathcal{F})$. Taking into account that in this case $G / \mathbf{S}(G) \cong \operatorname{Aut}_{G}(\mathbf{S}(G))$, we infer that

$$
G \in \mathcal{E}_{q} \varphi_{q}(\mathcal{F}) \subseteq \mathcal{E}_{q} \Phi_{L}(\mathcal{F})^{q}=\Phi_{L}(\mathcal{F})^{q} \subseteq \Phi_{L}(\mathcal{F})
$$

REMARK 3.7. Combining the preceding corollary with 1.6 , it is easily deduced that for any local formation $\mathcal{F}$,

$$
\Phi_{X[\mathcal{P}\}}(\mathcal{F}) \cap \mathcal{P}_{I} \subseteq \Phi_{L}(\mathcal{F}),
$$

where $\mathcal{P}, \mathcal{P}_{I}$ denote, respectively, the classes of primitive groups and of primitive groups with an abelian minimal normal subgroup. 
Clearly, for classes $\mathcal{K}$ consisting of soluble groups only, the closure operator $C_{\mathrm{Sch}}=$ $X_{\mathcal{P}} Q=X[\mathcal{P}]$ (which associates to each class the smallest Schunck class containing it) associates to $\mathcal{K}$ the same class as $X\left[\mathcal{P}_{I}\right]=X_{\mathcal{P}_{I}} Q$; so for local formations $\mathcal{F}$ of soluble groups we obtain from the last observation:

$$
\Phi_{C_{\text {Sch }}}(\mathcal{F})=\Phi_{X\left[\mathcal{P}_{I}\right]}(\mathcal{F})=X\left[\mathcal{P}_{I}\right]\left(\Phi_{X\left[\mathcal{P}_{I}\right]}(\mathcal{F}) \cap \mathcal{P}_{I}\right) \subseteq C_{\mathrm{Sch}} \Phi_{L}(\mathcal{F})=\Phi_{L}(\mathcal{F})
$$

(It is worthwhile to note that for local formations $\mathcal{F}$ of soluble groups the last condition in the definition of $\Psi_{p}(\mathcal{F})$ always holds, simplifying that definition considerably. Incidentally, it is also easy to see that for all local formations $\mathcal{F}$ of arbitrary finite groups

$$
\begin{gathered}
\Phi_{C_{\mathrm{Sch}}}(\mathcal{F}) \supseteq \bigcup_{p \in \mathrm{P}}\left\{(H / K) *(G / K) \mid G \in \mathcal{F}_{q} \cap \mathcal{M} \text { for some } q \in \chi(\mathcal{F}) \backslash\{p\}\right. \\
H / K \text { chief factor of } G \text { with } p \in \pi(H / K)\} .)
\end{gathered}
$$

Furthermore, note that on the other hand $\Phi_{L}(\mathcal{F}) \subseteq \mathcal{F}=\Phi_{Q R_{0}}(\mathcal{F})$ by 2.2a for all local formations $\mathcal{F}$.

In 2.2b we have obtained the inclusion $\mathcal{F} \subseteq \Phi_{Q R_{0}}(\mathcal{F})_{\mathbf{s}}$ for all formations $\mathcal{F}$ other than $\{1\}$. A weak version of this statement is:

$$
\mathcal{F} \subseteq\left(D_{0} \mathcal{X}_{0}\right) \Phi_{Q R_{0}}(\mathcal{F})=\left(Q R_{0} \mathcal{X}_{0}\right) \Phi_{Q R_{0}}(\mathcal{F})
$$

where $\mathcal{X}_{0}$ denotes the class of all simple groups. Our last corollary will provide an analogue to this assertion for $L$ instead of $Q R_{0}$.

CoROLLaRY 3.8. $\mathcal{F} \subseteq\left(L \mathcal{X}_{0}\right) \Phi_{L}(\mathcal{F})$ for any local formation $\mathcal{F} \neq\{1\}$.

Proof: By the main result of $[16],\left(L \mathcal{X}_{0}\right) \Phi_{L}(\mathcal{F})$ is a local formation, and as such it is a Schunck class. To establish the inclusion $\mathcal{F} \subseteq\left(L \mathcal{X}_{0}\right) \Phi_{L}(\mathcal{F})$, it therefore suffices to show that every monolithic primitive group $G \in \mathcal{F}$ belongs to $\left(L \mathcal{X}_{0}\right) \Phi_{L}(\mathcal{F})$.

First assume that $\mathbf{S}(G)$ is non-abelian. By $2.2 \mathrm{a} G / \mathrm{S}(G) \in \Phi_{Q R_{0}}\left(\mathcal{F}_{p}\right)$ for any prime $p$ dividing $|\mathbf{S}(G)|$, for in this case $G \in \mathcal{F}_{p}$. Now 3.1 yields that

$$
G / S(G) \in \Phi_{L}(\mathcal{F})^{p} \subseteq \Phi_{L}(\mathcal{F})
$$

and as $\mathbf{S}(G) \in D_{0} \mathcal{X}_{0} \subseteq L \mathcal{X}_{0}$ holds trivially, in this case we are done.

For the remainder of the proof we may therefore assume that $S(G)$ is an elementary abelian p-group for some $p \in \chi(\mathcal{F})$. Then $G^{*}=G / \mathbf{S}(G) \cong \operatorname{Aut}_{G}(\mathbf{S}(G)) \in \mathcal{F}_{p}$. Furthermore, without loss of generality, $G^{*} \in \mathcal{M}$ (where the trivial case when $G^{*}=1$ is being ignored): 
In fact, we aim at showing that either

$$
G^{*} \in \Phi_{L}(\mathcal{F})
$$

or else there exists some $S^{*} \unlhd G^{*}$ with $1 \neq S^{*} \leqslant \mathrm{~S}\left(G^{*}\right), G^{*} / S^{*} \in \Phi_{L}(\mathcal{F})$ and $p|| T^{*} \mid$ for every minimal normal subgroup $T^{*}$ of $G^{*}$ contained in $S^{*}$; yet it suffices to prove this for a set $\left\{G^{*} / N_{1}^{*}, \ldots, G^{*} / N_{n}^{*}\right\}$ of monolithic quotients $G^{*} / N_{i}^{*}$ of $G^{*}$ satisfying $\bigcap_{i=1}^{n} N_{i}^{*}=1$, provided only we choose this set such that none of the $N_{i}^{*}$ is redundant, for in that case $\mathrm{S}\left(G^{*} / N_{i}^{*}\right)$ is a minimal normal subgroup of $G^{*}$ (to within $G^{*}$-isomorphism) - more precisely,

$$
\mathbf{S}\left(G^{*}\right)=\bigcap_{i=1}^{n} \mathbf{S}\left(G^{*} \div N_{i}^{*}\right)=\times_{i \div 1}^{n}\left(\mathrm{~S}\left(G^{*} \div N_{i}^{*}\right) \cap \bigcap_{i \neq j=1}^{n} N_{j}^{*}\right) .
$$

Here it has to be noted that the groups $G^{*} / N_{i}^{*}$ are also of the form $\operatorname{Aut}_{\bar{G}_{i}}\left(\mathbf{S}\left(G_{i}\right)\right)$ for suitable monolithic primitive groups $\bar{G}_{i}$ (for example, take the semidirect product of $G^{*} / N_{i}^{*}$ with an irreducible and faithful $G^{*} / N_{i}^{*}$-module over $\left.G F(p)\right)$ : the case that $\mathrm{O}_{p}\left(G^{*} / N_{i}^{*}\right) \neq 1$ is excluded by the minimal choice of the set $\left\{G^{*} / N_{1}^{*}, \ldots, G^{*} / N_{n}^{*}\right\}$, for $G^{*}=G / \mathbf{S}(G) \cong \operatorname{Aut}_{G}(\mathbf{S}(G))$.

Now that $G^{*} \in \mathcal{F}_{p} \cap \mathcal{M}$, we may apply 3.6 to get that $G^{*} \in \Phi_{L}(\mathcal{F})$, except, perhaps, when $\left(\Phi\left(G^{*}\right)=1\right.$ and) $\{p\} \subset \pi\left(S\left(G^{*}\right)\right)$. In the latter case, however, in the second paragraph of this proof we have seen that $G^{*} / \mathbf{S}\left(G^{*}\right) \in \Phi_{L}(\mathcal{F})$, so it remains to note that, in view of $G^{*} \in \mathcal{M},\{p\} \subset \pi\left(\mathbf{S}\left(G^{*}\right)\right)$ and $\mathbf{S}(G) \in \mathcal{E}_{p}$, here we have that $\mathbf{S}(G \div \mathbf{S}(G)) \in L \mathcal{X}_{0}$.

Finally, we show how to calculate more easily than in [12] the $L$-Frattini class of some specific local formations by applying 3.1. The point here, of course, is that 3.1 describes a local definition of that Frattini class, as opposed to the "lower and upper bounds" obtained in [12].

EXAMPLES 3.9: (1) For $\mathcal{F}=L\left\{S_{4}\right\}$ one checks that

$$
\mathcal{F}_{2}=Q R_{0}\left\{S_{3}\right\}, \mathcal{F}_{3}=Q R_{0}\left\{Z_{2}\right\}, \mathcal{F}_{p}=\emptyset \text { for all primes } p \notin\{2,3\}
$$

and

$$
\varphi_{2}(\mathcal{F})=\{1\}, \varphi_{3}(\mathcal{F})=\mathcal{F}_{3}
$$

so 3.1 gives that

$$
\Phi_{Q R_{0}}\left(\mathcal{F}_{2} \div \varphi_{2}(\mathcal{F})\right)=\Phi_{Q R_{0}}\left(Q R_{0}\left\{S_{3}\right\}\right)=Q R_{0}\left\{Z_{2}\right\} \supset \Phi_{L}(\mathcal{F})_{2}=\{1\}=\varphi_{2}(\mathcal{F})
$$

in particular $\quad \Phi_{L}(\mathcal{F})^{2}=\mathcal{E}_{2} \supset Q R_{0}\left\{Z_{2}\right\}=\Phi_{Q R_{0}}\left(\mathcal{F}_{2} \div \varphi_{2}(\mathcal{F})\right)$,

$$
\Phi_{Q R_{0}}\left(\mathcal{F}_{3} \div \varphi_{3}(\mathcal{F})\right)=\Phi_{Q R_{0}}\left(\mathcal{F}_{3} \div \mathcal{F}_{3}\right)=\mathcal{F}_{3}=\Phi_{L}(\mathcal{F})_{3} \supset \Phi_{Q R_{0}}\left(\mathcal{F}_{3}\right)=\{1\}
$$


(Note that here $\Phi_{L}(\mathcal{F})_{p}=\varphi_{p}(\mathcal{F})$ for all primes $p$. ) It follows that

$$
\Phi_{L}(\mathcal{F})=L\left\{S_{3}\right\}
$$

(2) 3.4 applies to $\mathcal{F}=L\left\{S_{3}\right\}$, with

$$
\mathcal{F}_{3}=Q R_{0}\left\{Z_{2}\right\}, \varphi_{3}(\mathcal{F})=\emptyset, \text { yet } \Phi_{L}(\mathcal{F})_{3}=\Phi_{Q R_{0}}\left(\mathcal{F}_{3}\right)=\{1\}
$$

and

$$
\Phi_{L}(\mathcal{F})_{2}=\{1\} \text {, so } \Phi_{L}(\mathcal{F})=\mathcal{E}_{2} \times \mathcal{E}_{3} \text {. }
$$

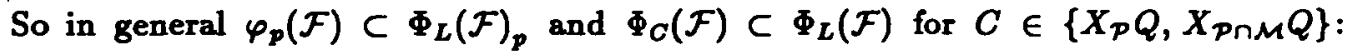
observe that (by 1.6) $\Phi_{C}(\mathcal{F})=\mathcal{E}_{2}$. It is also interesting to note that

$$
L\left\{S_{4} / \mathbf{S}\left(S_{4}\right)\right\}=L\left\{S_{3}\right\}=\Phi_{L}\left(L\left\{S_{4}\right\}\right) \lessdot_{L} L\left\{S_{4}\right\},
$$

whereas

$$
L\left\{S_{3} / \mathbf{S}\left(S_{3}\right)\right\}=L\left\{Z_{2}\right\}=\mathcal{E}_{2} \subset \Phi_{L}\left(L\left\{S_{3}\right\}\right) \ll_{L} L\left\{S_{3}\right\} .
$$

(3) The minimal local definition of the formation $\mathcal{N A}$ is given by

$$
(\mathcal{N A})_{p}=\mathcal{A}_{p^{\prime}} \text { for all primes } p \text {. }
$$

Since it is evident from $2.2 \mathrm{a}$ that $\Phi_{Q R_{0}}\left(\mathcal{A}_{p^{\prime}}\right)=\mathcal{A}_{p^{\prime}}, 3.4$ yields that

so

$$
\Phi_{L}(\mathcal{N} \mathcal{A})_{p}=\Phi_{Q R_{0}}\left((\mathcal{N A})_{p}\right)=\Phi_{Q R_{0}}\left(\mathcal{A}_{p^{\prime}}\right)=\mathcal{A}_{p^{\prime}}=(\mathcal{N A})_{p}
$$

$$
\Phi_{L}(\mathcal{N A})=\mathcal{N} \mathcal{A} \text {. }
$$

(4) The minimal local definition of the formation $\bar{U}$ of supersoluble groups is given by

$$
\ddot{u}_{p}=\{G \in \mathcal{A}|\exp (G)| p-1\} \text { for all primes } p .
$$

For odd primes $p$, let $p_{i}, i=1, \ldots, n_{p}$ denote the primes dividing $p-1$. As any formation of abelian groups is generated by the cyclic groups contained in it, it is clear that the formations

$$
\ddot{\mathcal{U}}_{p, i}=\left\{G \in \mathcal{A}|\exp (G)|(p-1) / p_{i}\right\}, i=1, \ldots, n_{p},
$$

are precisely the maximal subformations of $\ddot{\mathcal{u}}_{p}$. Hence

$$
\Phi_{Q R_{0}}\left(\ddot{U}_{p}\right)=\left\{G \in \mathcal{A}|\exp (G)|(p-1) / p_{1} \ldots p_{n_{p}}\right\} .
$$

By 3.4, it follows that

$$
\Phi_{L}(\bar{U})_{2}=\{1\} \text { and } \Phi_{L}(\bar{U})_{p}=\Phi_{Q R_{0}}\left(\bar{U}_{p}\right), p \neq 2 ;
$$

in particular

$$
\Phi_{L}(\ddot{u})<_{L} \ddot{u} \text {. }
$$


(5) Let $\mathcal{F}=L\{E\}$ where $E$ is any non-abelian simple group. Then obviously $\mathcal{F}_{p}=Q R_{0}\{E\}=D_{0}\{E\}$ and $\varphi_{p}(\mathcal{F})=\emptyset$ for all $p \in \chi(\mathcal{F})=\pi(E)$. As $\mathcal{F}_{p}=D_{0}\{E\}$, we see that $\Phi_{Q R_{0}}\left(\mathcal{F}_{p}\right)=\{1\}$, whence

$$
\Phi_{Q R_{0}}\left(\mathcal{F}_{p} \div \varphi_{p}(\mathcal{F})\right)=\{1\}
$$

and thus

$$
\Phi_{L}(\mathcal{F})_{p}=\{1\} \text { for all } p \in \chi(\mathcal{F})=\pi(E) \text {. }
$$

Consequently

$$
\Phi_{L}(\mathcal{F})=\mathcal{N}_{\pi(E)} \text {. }
$$

Note that $E \in\left(\bigcap_{p \in \pi(E)} \mathcal{F}_{p}\right) \backslash \Phi_{L}(\mathcal{F}):$ this shows that the hypotheses imposed on $\mathrm{S}(G)$ in 3.6 are not redundant.

(6) Let $\mathcal{F}_{i}=\mathcal{N} \mathcal{G}_{i}$ where $\mathcal{G}_{i}=Q R_{0} \mathcal{G}_{i} \subseteq \mathcal{N}, i=1$, 2. Then $\left(\mathcal{F}_{i}\right)_{p}=\mathcal{G}_{i} \cap \mathcal{E}_{p^{\prime}}$ for all primes $p$. Choosing the $\mathcal{G}_{1}$ and $\mathcal{G}_{2}$ such that $\{1\} \subset \mathcal{G}_{1} \subset \mathcal{G}_{2} \subseteq \mathcal{E}_{p}$ and $\Phi_{Q R_{0}}\left(\mathcal{G}_{2}\right)=$ $\mathcal{G}_{1}=\Phi_{Q R_{0}}\left(\mathcal{G}_{1}\right)$ yields local formations $\mathcal{F}_{i}$ with $\mathcal{F}_{1} \subset \mathcal{F}_{2}$, but $\Phi_{L}\left(\mathcal{F}_{2}\right)=\mathcal{F}_{1}=\Phi_{L}\left(\mathcal{F}_{1}\right)$.

\section{REFERENCES}

[1] D.W. Barnes and O.H. Kegel, 'Gaschütz functors on finite soluble groups', Math. Z. 94 (1966), 134-142.

[2] R.M. Bryant, R.A. Bryce and B. Hartley, 'The formation generated by a finite group.', Bull. Austral. Math. Soc. 2 (1970), 347-357.

[3] P. Förster, 'Closure operations for Schunck classes and formations of finite solvable groups', Math. Proc. Cambridge Philos. Soc. 85 (1979), 253-259.

[4] P. Förster, 'Projektive Klassen endlicher Gruppen I. Schunck- und Gaschützklassen', Math. Z. 186 (1984), 149-178.

[5] P. Förster, 'Projektive Klassen endlicher Gruppen Ila. Gesättigte Formationen: Ein allgemeiner Satz vom Gaschütz-Lubeseder-Baer-Typ', Publ. Sec. Mat. Univ. Autònoma Barcelona 28 (1985), 39-76.

[6] P. Förster, 'An elementary proof of Lubeseder's theorem', Arch. Math. 52 (1989), 417-419.

[7] P. Förster and E. Salomon, 'Local definitions of local homomorphs and formations of finite groups', Bull. Austral. Math. Soc. 31 (1985), 5-34.

[8] P. Hall, 'On non-strictly simple groups', Proc. Cambridge Philos. Soc. 59 (1963), 531-553.

[9] U.C. Herzfeld, 'Frattiniklassen und maximale Teilklassen, insbesondere zu Formationen endlicher Gruppen', Dissertation, Mainz (1985).

[10] U.C. Herzfeld, 'Frattiniklassen und maximale Teilklassen, insbesondere zu Formationen endlicher Gruppen', Dissertation, Mainz (1986).

[11] U.C. Herzfeld, 'Frattiniclasses and maximal subclasses, in particular of formations of finite groups', J. Algebra 117 (1988), 93-98.

[12] U.C. Herzfeld, 'Frattiniclasses of formations of finite groups', Boll. Un. Mat. Ital. (7) 2-B (1988), 601-611. 
[13] B. Huppert, Endliche Gruppen I (Springer-Verlag, Berlin, Heidelberg, New York, 1967).

[14] L.G. Kovács and M.F. Newman, 'On critical groups', J. Austral. Math. Soc. 6 (1966), 237-250.

[15] H. Neumann, Varieties of groups (Springer-Verlag, Berlin, Heidelberg, New York, 1967).

[16] E. Salomon, 'Local definability of formation products', Comm. Algebra (to appear).

Mathematics Research Section, IAS

The Australian National University

Canberra ACT 2601

Australis
Current address:

Fachbereich Mathematik

J. Gutenberg-Universität

D-6500 Mains 1

Federal Republic of Germany 